\title{
KAWASAKI DISEASE IN INFANTS LESS THAN ONE YEAR OF AGE: AN ITALIAN COHORT
}

\author{
G. Mastrangelo 1 , S. Trapani², D. Lasagni², G. Calabri ${ }^{3}$, M. Resti², R. Cimaz ${ }^{4}$
}

${ }^{1}$ Pediatric Residency program, Meyer Children's Hospital, University of Florence, Florence, Italy , ${ }^{2}$ Department of Pediatrics, Meyer Children's Hospital, University of Florence, Florence, Italy, ${ }^{3}$ Cardiology Unit, Meyer Children's Hospital, University of Florence, Florence, Italy, ${ }^{4}$ Rheumatology Unit, Meyer Children's Hospital, University of Florence, Florence, Italy

\section{OBJECTIVES}

1. To analyze clinical and laboratory features of $K D$ among an Italian cohort of infants below 12 months.

2. To confirm whether infants with incomplete $K D$ have a more severe disease and a greater predilection for cardiac involvement.

\section{METHODS}

- Retrospective chart review of children diagnosed with KD between January 2008 and December 2017 admitted to Meyer Children Hospital in Florence, Italy.

- Data were analyzed by Epi Info Statistical Software version 7.1.5.2.

- Inclusion criteria: disease onset below 12 months of age according to AHA guidelines (including incomplete and atypical Kawasaki disease).

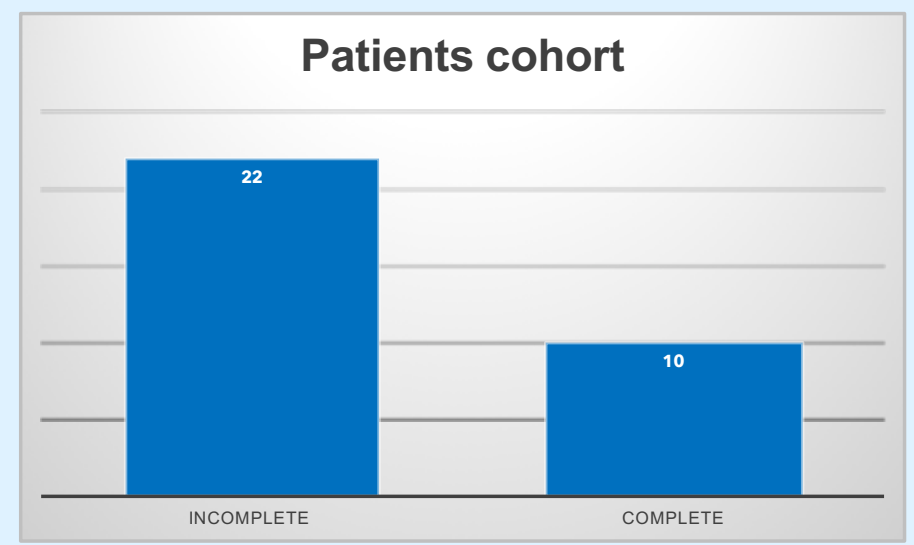

\section{Clinical Features}

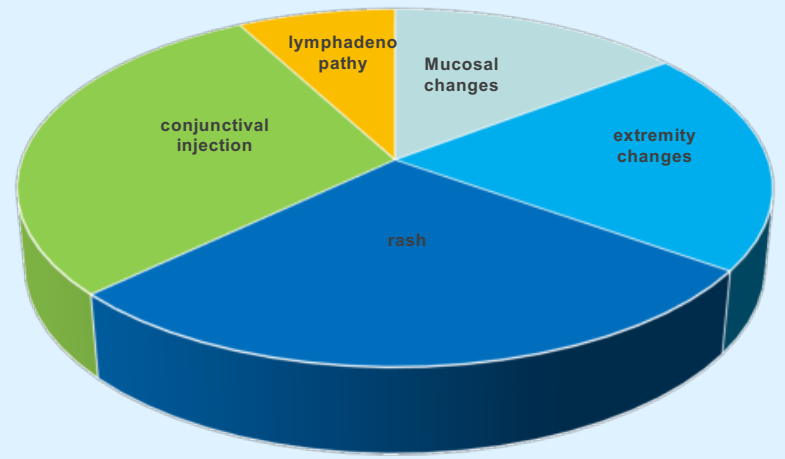

\section{RESULTS}

- 113 patients with KD of whom 32 infants belows 12 months of age $(28,3 \%)$. (M:F= 20:12).

- Mean age of presentation was 5,7 $\pm 2,7$ months. 19 patients $(59,4 \%)$ were less than 6 months of whom $8 / 32$ were below 3 months of age $(25 \%)$.

- Mucosal changes were present in 13 patients $(40,6 \%)$; extremity changes in 18 (56,2\%); rash in 25 (78,1\%); conjunctival injection in $26(81,2 \%)$ and cervical lymphadenopathy in 7 (21,8 \%).

- 22 patients $(68,7 \%)$ had incomplete KD.

- Heart involvement was present in $19(59,4 \%)$ patients of whom $13(59 \%)$ had incomplete form.

- Coronary artery abnormalities (CAA) were detected in $16 / 32$ (50\%) patients of whom 12 had an incomplete form (75\%).

- The mean time for diagnosis was 7 days (range 3-22) and it was longer in the incomplete KD $(7,6$ VS 5,8).

- PCR and platelets values were all higher in complete KD than in incomplete forms, without any significant difference between the two groups.

- ESR was higher in complete forms than in incomplete with a statistical significative difference $(p<0,01)$

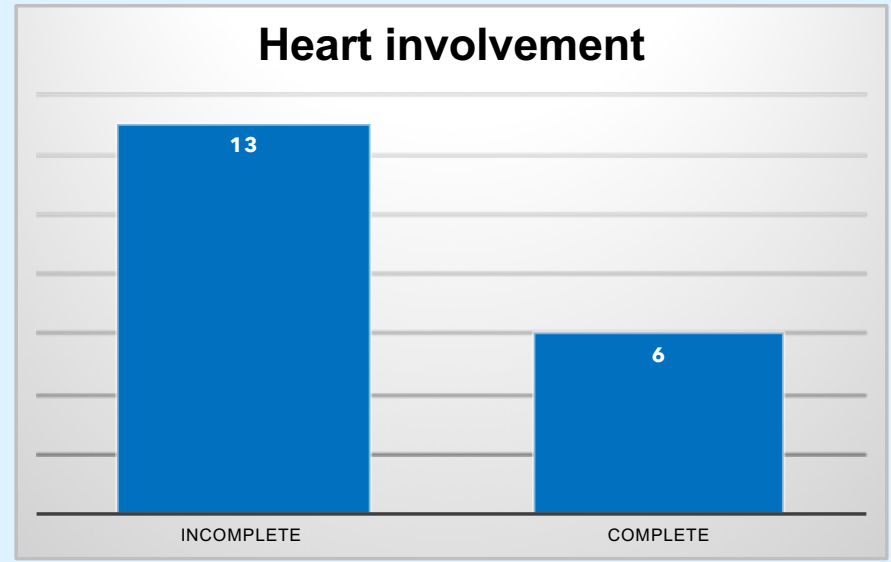

\section{CONCLUSION:}

- Incomplete KD was more commonly found in children younger than one year

- Incomplete forms were diagnosed later than the complete ones

- Infants with incomplete KD have a more severe disease and a greater predilection for cardiac involvement. 